University of Zurich

Department of Economics

Working Paper Series

ISSN 1664-7041 (print)

ISSN1664-705X(online)

Working Paper No. 46

\title{
Identification and estimation of thresholds in the fixed effects ordered logit model
}

Gregori Baetschmann

November 2011 


\title{
Identification and estimation of thresholds in the fixed effects ordered logit model
}

\author{
Gregori Baetschmann* \\ University of Zurich, Department of Economics
}

This version: November 11, 2011

\begin{abstract}
The paper proposes a new estimator for the fixed effects ordered logit model. In contrast to existing methods, the new procedure allows estimating the thresholds. The empirical relevance and simplicity of implementation is illustrated in an application to the effect of unemployment on life satisfaction.
\end{abstract}

Keywords: ordered response, panel data, correlated heterogeneity, incidental parameters

JEL Codes: C23, C25, J28, J64

* I thank Kevin Staub and Rainer Winkelmann for very valuable comments.

Address for correspondence: University of Zurich, Department of Economics, Zürichbergstr. 14, CH-8032 Zürich, Switzerland, శ+41 44634 2295, gregori.baetschmann@econ.uzh.ch 


\section{Introduction}

There exist a number of proposals in the literature on how to estimate a panel-ordered logit model with individual fixed effects - Das and van Soest (1999), Ferrer-i-Carbonell and Frijters (2004) and most recently Baetschmann, Staub and Winkelmann (2011). A drawback of all these estimators is that they do not identify the threshold parameters. This paper proposes a new estimating procedure which allows estimating these thresholds. Knowing the thresholds has three advantages: First, the thresholds are helpful for interpreting the regression coefficients; second, they make it possible to obtain statements about the effect of a changing $x$ on the observed ordered variable and not only on the latent variable; and third, comparing the differences between the thresholds can be interesting in itself. The new procedure can be easily implemented using existing software for conditional maximum likelihood (CML) logit estimation with cluster corrected standard errors.

The paper proceeds as follows. Section 2 presents the fixed effects ordered logit model and discusses the new estimation procedure. In section 3 the new estimator is applied to data from the German Socioeconomic Panel.

\section{Econometric Methods}

\subsection{The FE ordered logit model}

The fixed effects ordered logit model relates the latent variable $y_{i t}^{*}$ for individual $i$ at time $t$ to a linear index of observable characteristics $x_{i t}$ and unobservable characteristics $\alpha_{i}$ and $\varepsilon_{i t}:$

$$
y_{i t}^{*}=x_{i t}^{\prime} \beta+\alpha_{i}+\varepsilon_{i t}, \quad i=1, \ldots, N \quad t=1, \ldots, T .
$$

The time-invariant part of the unobservables $\left(\alpha_{i}\right)$, called fixed effect, can be statistically dependent of $x_{i t}$. 
The latent variable is tied to the (observed) ordered variable $y_{i t}$ by the observation rule:

$$
y_{i t}=k \quad \text { if } \quad \tau_{k}<y_{i t}^{*} \leq \tau_{k+1}, \quad k=1, \ldots, K
$$

where the thresholds $\tau$ are assumed to be strictly increasing $\left(\tau_{k}<\tau_{k+1} \forall k\right)$ and $\tau_{1}=-\infty$, $\tau_{K+1}=\infty$

The specification of the fixed effects ordered logit model is completed by assuming that the $\varepsilon_{i t}$ are conditionally independent and identically standard logistically distributed. I.e., if $F(\cdot)$ denotes the cdf

$$
F\left(\varepsilon_{i t} \mid x, \alpha\right)=\frac{\exp \left(\varepsilon_{i t}\right)}{1+\exp \left(\varepsilon_{i t}\right)} \equiv \Lambda\left(\varepsilon_{i t}\right)
$$

Hence, the probability of observing an outcome equal to $k$ for individual $i$ at time $t$ using (??), (??) and (??) can be written as

$$
\operatorname{Pr}\left(y_{i t}=k \mid x_{i t}, \alpha_{i}\right)=\Lambda\left(\tau_{k+1}-x_{i t}^{\prime} \beta-\alpha_{i}\right)-\Lambda\left(\tau_{k}-x_{i t}^{\prime} \beta-\alpha_{i}\right)
$$

whereas the probability of an outcome greater or equal to $k$ is

$$
\operatorname{Pr}\left(y_{i t} \geq k \mid x_{i t}, \alpha_{i}\right)=\Lambda\left(x_{i t}^{\prime} \beta+\alpha_{i}-\tau_{k}\right)
$$

Equation (??) and (??) show that the location of the $\tau$ 's and $\alpha$ 's cannot be distinguished. Thus the constant and the second threshold $\left(\tau_{2}\right)$ are normalized to zero.

The problem with maximum likelihood estimation based on (??) is that the expression depends on the individual fixed effect $\alpha_{i}$. Including individual dummies in the estimation procedure to account for fixed effects is not a solution due to the "incidental parameter problem" - e.g. Chamberlain (1980).

\subsection{Illustration of the estimation procedure}

The binary logit model is one of the few nonlinear models, where it is known how to deal with fixed effects. For this model, Chamberlain (1980) proposed to condition the likelihood 
on the number of one's in individual's record to get rid of the individual fixed effects. Chamberlain's method can be applied to the ordered logit model as well. The procedure is as follows: First, the ordered dependent variable $y$ is dichotomized to a binary one. The binary variable is denoted by $d$ and the cutoff by $k: d=\mathbb{1}(y \geq k)$, where $\mathbb{1}()$ is the indicator function. Second, Chamberlain's estimation procedure is applied to $d$.

To illustrate this procedure consider an individual which is observed two times, where the first observation equals 3 and the second equals 1: $y_{1}=3, y_{2}=1$. We assume that the variable $y$ can take the values 1, 2 and 3, thus $k$ can be either 2 or 3 . In this example both choices result in the same binary dependent variable, $d_{1}=1$ and $d_{2}=0$. The following conditional probability results:

$$
\begin{aligned}
\operatorname{Pr} & {\left[d_{1}=1 \cap d_{2}=0 \mid\left(d_{1}=1 \cap d_{2}=0\right) \cup\left(d_{1}=0 \cap d_{2}=1\right)\right] } \\
& =\operatorname{Pr}\left[y_{1} \geq k \cap y_{2}<k \mid\left(y_{1} \geq k \cap y_{2}<k\right) \cup\left(y_{1}<k \cap y_{2} \geq k\right)\right] \\
& =\frac{\frac{\exp \left(x_{1}^{\prime} \beta+\alpha-\tau_{k}\right)}{1+\exp \left(x_{1}^{\prime} \beta+\alpha-\tau_{k}\right)} \frac{1}{1+\exp \left(x_{2}^{\prime} \beta+\alpha-\tau_{k}\right)}}{\frac{\exp \left(x_{1}^{\prime} \beta+\alpha-\tau_{k}\right)}{1+\exp \left(x_{1}^{\prime} \beta+\alpha-\tau_{k}\right)} \frac{1}{1+\exp \left(x_{2}^{\prime} \beta+\alpha-\tau_{k}\right)}+\frac{\exp \left(x_{2}^{\prime} \beta+\alpha-\tau_{k}\right)}{1+\exp \left(x_{1}^{\prime} \beta+\alpha-\tau_{k}\right)}} \\
& =\frac{\exp \left(x_{1}^{\prime} \beta+\alpha-\tau_{k}\right)}{\exp \left(x_{1}^{\prime} \beta+\alpha-\tau_{k}\right)+\exp \left(x_{2}^{\prime} \beta+\alpha-\tau_{k} \beta+\tau_{k}\right)}=\frac{\exp \left(x_{1}^{\prime} \beta\right)}{\exp \left(x_{1}^{\prime} \beta\right)+\exp \left(x_{2}^{\prime} \beta\right)} .
\end{aligned}
$$

The last expression in (??) is independent of $\alpha$. Thus $\beta$ can be estimated by conditional maximum likelihood.

The problem with this procedure is that the $\tau$ 's disappear from the probability expression as well and are therefore not identified. The reason is that the same cutoff is used for all observations of an individual, so there is no "cutoff-variation" within a conditional likelihood contribution. By contrast, if the observations of an individual are dichotomized at different cutoff points and the probability expression is applied accordingly, the thresholds are identified. Consider again the above example, but suppose now that the first observation is dichotomized at 2 and the second at 3 . The probability that the the first dichotomization is one, given that either the first or the second is one (but not both) is 
now:

$$
\begin{aligned}
\operatorname{Pr} & {\left[y_{1} \geq 2 \cap y_{2}<3 \mid\left(y_{1} \geq 2 \cap y_{2}<3\right) \cup\left(y_{1}<2 \cap y_{2} \geq 3\right)\right] } \\
& =\frac{\frac{\exp \left(x_{1}^{\prime} \beta+\alpha-\tau_{2}\right)}{1+\exp \left(x_{1}^{\prime} \beta+\alpha-\tau_{2}\right)} \frac{1}{1+\exp \left(x_{2}^{\prime} \beta+\alpha-\tau_{3}\right)}}{\frac{\exp \left(x_{1}^{\prime} \beta+\alpha-\tau_{2}\right)}{1+\exp \left(x_{1}^{\prime} \beta+\alpha-\tau_{2}\right)} \frac{1}{1+\exp \left(x_{2}^{\prime} \beta+\alpha-\tau_{3}\right)}+\frac{1}{1+\exp \left(x_{1}^{\prime} \beta+\alpha-\tau_{2}\right)} \frac{\exp \left(x_{2}^{\prime} \beta+\alpha-\tau_{3}\right)}{1+\exp \left(x_{2}^{\prime} \beta+\alpha-\tau_{3}\right)}} \\
& =\frac{\exp \left(x_{1}^{\prime} \beta+\alpha-\tau_{2}\right)}{\exp \left(x_{1}^{\prime} \beta+\alpha-\tau_{2}\right)+\exp \left(x_{2}^{\prime} \beta+\alpha-\tau_{3}\right)}=\frac{\exp \left(x_{1}^{\prime} \beta-\tau_{2}\right)}{\exp \left(x_{1}^{\prime} \beta-\tau_{2}\right)+\exp \left(x_{2}^{\prime} \beta-\tau_{3}\right)} .
\end{aligned}
$$

This expression is independent of $\alpha$ but depends on $\beta$ and the $\tau$ 's. Hence there is no incidental parameter problem, and $\beta$ and $\tau$ can be estimated by maximum likelihood. The method can easily be generalized to situations with more than two time periods and more than two possible cutoff points.

\subsection{Practical implementation - choosing the cutoff points}

The question arises, which combinations of observation specific cutoff points to include in the estimation procedure. One possibility is to include all feasible combinations. Cluster standard errors can be used to account for the dependence between the conditional likelihood contributions of the same individual (White, 1982). The same idea of including more than one "clone" of an individual combined with cluster standard errors is used by the BUC estimator (Baetschmann, Staub and Winkelmann, 2011) to estimate $\beta$ in the FE ordered logit model with individual specific thresholds. In the previous example, there are four combinations: Both observations can be dichotomized at two cutoff points and all four combinations are possible. Among those, only the two combinations with different cutoff points are informative for estimating the $\tau$ 's.

The inclusion of all possible cutoff point combinations in the estimation procedure is only feasible if the number of time periods $(T)$ and the number of categories $(K)$ of the dependent variable are small, because the number of possible combinations is $(K-1)^{T}$. For example if $T$ and $K$ are equal to 10, there exist more than three billion possible copies of each individual. Often the researcher is more interested in estimating $\beta$ than $\tau$. On 
this account, I propose to include all clones with no variation in the cutoff to estimate $\beta$ precisely and fill up the rest of the dataset with a limited number of clones with random variation in the cutoff points. (Stata code is available from the author upon request.)

\section{Illustration}

To illustrate the estimation procedure, the new fixed effects ordered logit estimator is applied to the model and dataset of Winkelmann and Winkelmann (1998). The dataset consists of a sample from the German Socioeconomic Panel going from 1984 to 1989 with 4'261 individuals. The dependent variable is satisfaction with life, which is measured as answer to the question "How satisfied are you at present with your life as a whole?". The answers ranges from 0, "completely dissatisfied", to 10, "completely satisfied". To be consistent with the notation of the theoretical part of this paper, the dependent variable is recoded and ranges now from 1 to 11.

If each individual would be dichotomized in all possible ways, the resulting dataset would consist of more than four billion entries. Hence I decided to include all clones with a constant cutoff to estimate $\beta$ precisely, plus ten clones of each individual, whose observations are dichotomized at observation specific random cutoff points. Compared to other proposed estimators, individuals without variation in the ordered dependent variable are not automatically excluded from the estimation procedure. The reason is that variation in the ordered dependent variable $(y)$ is not a precondition for variation in the dichotomized dependent variable $(d)$ if varying cutoffs within a conditional likelihood contribution are allowed.

The columns with heading " $y \geq 8$ " of table (1) show the estimates of the Chamberlain "estimator" if the ordered variable is dichotomized at 8. These estimates are also reported in Winkelmann and Winkelmann (1998). The results of the new estimation procedure are very similar and are listed in the columns with heading " $\mathrm{BUC}_{\tau}$ ". The standard errors 
of the new estimator are slightly smaller. The essential advantage of the new estimation procedure is that estimates for the thresholds are available. The differences between them ranges from 0.66 between $\tau_{3}$ and $\tau_{4}$ to 1.69 between $\tau_{9}$ and $\tau_{10}$. Roughly speaking, the differences between the thresholds increase with the threshold number. This means that the effect of an increasing latent index - for example, by +1.10 when being employed rather than unemployed - on the ordered life satisfaction variable is largest for people with a low life satisfaction level. 


\section{References}

Baetschmann, G., Staub, K., Winkelmann, R., 2011. Consistent estimation of the fixed effects ordered logit model. IZA Discussion Paper No. 5443.

Das, M., van Soest, A., 1999. A panel data model for subjective information on household income growth. Journal of Economic Behavior \& Organization 40 (4), 409-426.

Chamberlain, G., 1980. Analysis of covariance with qualitative data. Review of Economic Studies 47 (1), 225-238.

Ferrer-i-Carbonell, A., and Frijters, P., 2004. How important is methodology for the estimates of the determinants of happiness? Economic Journal 114 (497), 641-659.

White, H., 1982. Maximum likelihood estimation of misspecified models. Econometrica 50 (1), 1-25.

Winkelmann, L., Winkelmann, R., 1998. Why are the unemployed so unhappy? Evidence from panel data. Economica 65 (257), 1-15. 